\title{
Einleitung: Das Haus nach seinem Ende
}

Auf dem »Haus « lasten Hypotheken. Wer sich mit diesem Begriff der Literaturund Kulturgeschichte des 19. Jahrhunderts nähern wollte, musste lange Zeit unvermeidlich auf jene Konzeption eines "ganzen Hauses « Bezug nehmen, die auf den Volkskundler und Sozialpolitiker Wilhelm Heinrich Riehl zurückgeht ${ }^{1}$ und ihre einflussreichste Deutung und Neukonturierung durch den Historiker Otto Brunner erfahren hat. ${ }^{2}$ Riehls "ganzes Haus « ist durch die Einheit von Haushalt, Familie und Betrieb gekennzeichnet und verweist auf die Häuser der Bauern und Handwerker, die der Volkskundler Mitte des 19. Jahrhunderts auf seinen Wanderungen durch das Land besucht und erforscht hatte. Es sind diese Häuser mit ihren Vorhöfen, geschmückten Türen und Fenstern und geräumigen Küchen, die Riehl den Städtebauern als architektonische Vorlage empfiehlt und welche die modernen Mietskasernen mit ihren engen Treppenaufgängen und zellenartigen Zimmeranordnungen ersetzen sollen. Riehls Darstellung eines "ganzen Hauses" partizipiert damit nicht zuletzt an den zeitgenössischen Debatten zur "Wohnungsfrage ", in denen das Elend der Arbeiter und Proletarier zuallererst auf ihre miserable Behausung zurückgeführt wird. Bei diesem wissenschaftlichen Beitrag zur ssozialen Ethnographie von Deutschland handelt es sich also auch um eine sozialpolitische Intervention: Die sich im Zuge der Industrialisierung stellende »soziale Frage « beantwortet Riehl mit dem Entwurf einer Lebensform. In gewissem Maße mag diese zu seiner Zeit noch empirisch aufweisbar gewesen sein. Der idealisierende Zug ihrer Darstellung ist jedoch unverkennbar. Konzepte des »Hauses " das gilt nicht für das Riehl'sche allein - stehen zur gesellschaftlichen Wirklichkeit in einem spannungsreichen Verhältnis.

Tatsächlich war die von Riehl beschriebene Lebensform im Verschwinden begriffen. Darauf lässt u.a. die Gesetzgebung schließen: In Preußen etwa, darauf hat Reinhart Koselleck hingewiesen ${ }^{3}$, zielte sie um 1800 auf eine wirtschaftliche Liberalisierung, der die altständische Herrschaftseinheit des »Hauses « im Wege stand. Ein weiterer Beleg für das Ende des »Hauses « ist der Untergang einer als "Oikonomik « bekannten Anleitungsliteratur, die sich an die "Hausväter " richtete und die Kunst der Haushaltsführung in allen Hinsichten beschrieb. Zwar erschienen auch nach 1750 noch »Hausbücher «, doch richte-

\footnotetext{
${ }^{1}$ Wilhelm Heinrich Riehl, Die Familie, 2. Aufl., Stuttgart, Augsburg 1855.

${ }^{2}$ Otto Brunner, "Das 'ganze Haus` und die alteuropäische >Ökonomik`", in: ders., Neue Wege der Verfassungs- und Sozialgeschichte, 2., verm. Aufl., Göttingen 1968, 103-127.

${ }^{3}$ Vgl. Reinhart Koselleck, „Die Auflösung des Hauses als ständischer Herrschaftseinheit. Anmerkungen zum Rechtswandel von Haus, Familie und Gesinde in Preußen zwischen der Französischen Revolution und 1848 «, in: Neithard Bulst, Joseph Goy, Jochen Hoock (Hrsg.), Familie zwischen Tradition und Moderne. Studien zur Geschichte der Familie in Deutschland und Frankreich vom 16. bis zum 20. Jahrhundert, Göttingen 1981, 109-124.
}

DVjs 85. Jg., 2 (2011) 
ten diese sich zunehmend an "Hausmütter " und behandelten nur Teilaspekte einer vormals umfassenden Haushaltungslehre. Setzt man diese Daten in Bezug zur Geschichte der Literatur, ergibt sich ein überraschender Befund: Die Romane nämlich handeln zunehmend von häuslichen Lebensformen. Ein frühes Beispiel ist Julie ou La Nouvelle Héloïe. Tatsächlich ist bei Rousseau eine Transformation der älteren Hauswirtschaftslehre in schöne Literatur zu beobachten, die auf die Formierung einer nun politischen Ökonomie und deren ganz andere Rationalität reagiert.

Es ist Otto Brunners Verdienst, diesem neuen Wirtschaftswissen gegenüber die Andersheit der älteren Oikonomik hervorgehoben zu haben. Dass er den Zugang zu ihrer Geschichte durch eine Apologie des "ganzen Hauses « eröffnet hat, ist jedoch eine weitere Hypothek, die es zu berücksichtigen gilt. Hier wird nicht nur eine alternative Wirtschaftsgeschichte in Aussicht gestellt, die gegen eine autoteleologische Historisierung der Kapitalwirtschaft die Genealogie häuslicher Subsistenzwirtschaft zur Geltung bringt. Wie diejenige Riehls weist auch Brunners Konzeption des "Hauses « einen stark idealisierenden Zug auf. Anders als jene reagiert diese jedoch nicht primär auf wirtschaftliche Veränderungen ihrer Zeit. In die dreißiger Jahre zurückreichend ist sie nach dem Zweiten Weltkrieg im Zusammenhang eines restaurativen Neu-Humanismus zur Entfaltung gekommen, dessen Verhältnis zu Nazi-Herrschaft und Völkermord wohl nur in psychoanalytischen Begriffen zu fassen ist. Hier ist Hermann Rebels Empfehlung zu folgen, die »Re-Imaginationen « des oikos - die Brunner'sche ist nur die vorerst letzte in einer langen Reihe - auf soziale "Traumata " hin zu befragen, die ihnen zugrunde liegen, d.h. nicht zuletzt auch auf soziale Konflikte, die eine oikonomische Regimentalität selbst produziert, konzeptionell aber verdrängt. ${ }^{4}$

Als Leitkategorie für positivistische Forschung ist das "ganze Haus " also denkbar ungeeignet. Das konnte der historischen Sozialforschung nicht verborgen bleiben. Doch hat sie das Imaginäre des oikos nicht einzuordnen gewusst. Nachdem die Vorstellung einer autarken, in sich geschlossenen und patriarchalisch organisierten Wirtschafts- und Herrschaftseinheit eine Reihe ideologiekritischer Repliken provoziert hat, ist in der gegenwärtigen Forschung eine interessante Neuausrichtung der Fragen zu verzeichnen. ${ }^{5}$ Dies gilt zunächst für die

\footnotetext{
${ }^{4}$ Hermann Rebel, »Reimagining the Oikos. Austrian Cameralism in its Social Formation «, in: Jay O’Brien, William Roseberry (Hrsg.), Golden Ages, Dark Ages. Imagining the Past in Anthropology and History, Berkeley 1991, 48-80.

${ }^{5}$ Vgl. Georg Fertig, Äcker, Wirte, Gaben. Ländlicher Bodenmarkt und liberale Eigentumsordnung im Westfalen des 19. Jahrhunderts, Berlin 2007. Zur Neuausrichtung der Frage nach der Familienwirtschaft vgl. die Einleitung von Gerd Spittler zu Aleksandr V. Cajanov, Die Lehre von der bäuerlichen Wirtschaft. Versuch einer Theorie der Familienwirtschaft im Landbau [Nachdr. d. Ausg. Berlin, Parey 1923], Frankfurt a.M., New York 1987.
} 
Disziplinen der Sozial- und Mikrogeschichte und der Ethnologie. Das von Riehl und Brunner propagierte häusliche Wirtschaften wird darin nicht mehr als nostalgische, rechtskonservativ tendenziöse Fiktion abgetan. Es wird vielmehr nach den materiellen Bedingungen und der globalen Verbreitung von Haus- und Familienwirtschaft gefragt. Unbelastet von modernisierungstheoretischen Vorgaben wird das Themenspektrum der Industrialisierung, Urbanisierung und der Genese von Lohnarbeit um Fragen der Agrarökonomie und um die Anthropologie der "Bedürfnisse « und divergenter Formen von Arbeit erweitert. Verschiedene Formen bäuerlichen, handwerklichen oder proletarischen Wirtschaftens und der verwandtschaftlichen und nachbarschaftlichen Tauschökonomie werden nicht länger als defizitäre Formen des modernen marktorientierten Handels begriffen, sondern in ihrer jeweiligen Eigendynamik, ihrer spezifischen Stabilität oder Labilität beschrieben und erklärt.

Nun besteht kaum Anlass zu der Behauptung, die Literatur des 19. Jahrhunderts habe diesen "sozialrealistischen « Anspruch der neueren, ideologisch entkernten »Haus «-Forschung vorgreifend erfüllt. Vielmehr tritt gerade in den Romanen das idealisierende Moment besonders deutlich hervor - und wo sie wie Wilhelm Raabes idyllische Darstellung einer nachbarschaftlichen Solidargemeinschaft zum sozialpolitischen Konzept des Hauses erkennbar auf Abstand gehen, hat man es mit Gegen-Imaginationen zu tun. So ist es kein Zufall, dass die prominenten wissenschaftlichen Arbeiten zum Haus regelmäßig auf Romane verweisen. Das gilt schon für Riehls Die Familie, wo Jeremias Gotthelf lobend erwähnt wird. Das gilt weiterhin für Brunners viel zitierten Aufsatz "Das 'ganze Haus` und die alteuropäische `Ökonomik«", der sich auf Gustav Freytags Soll und Haben bezieht, wie für sein Buch Adeliges Landleben und europäischer Geist, in dem Stifters Nachsommer Erwähnung findet. Nicht anders nimmt Heinrich Schurtz' Studie Altersklassen und Männerbünde aus dem Jahr 1902, in der sich der Ethnologe mit dem Konzept des Männerhauses auseinandersetzt, unter anderem auf Raabes Roman Abu Telfan Bezug.

Den hier versammelten Beiträgen liegt die Annahme zugrunde, dass solche Bezugnahmen auch dort, wo sie lediglich der Illustration dienen sollen, einen imaginären Transfer zwischen Häuserromanen und volkskundlichen bzw. sozialgeschichtlichen Darstellungen des Hauses bezeugen. Das gespannte Verhältnis der Rede vom Haus zu seiner Realität ist jedoch älter als dieser Transfer zwischen » moderner « Wissenschaft und Literatur. Es besteht von ihren philosophischen Anfängen an - die wirkungsgeschichtlich bedeutendste, die aristotelische oikos-Lehre spiegelt ja nicht etwa reale Sozialverhältnisse, sondern reagiert in normativer Absicht auf eine rasche Verbreitung des geldvermittelten Tauschhandels. Im Zuge der Transformation der älteren Hauslehre ging auch dieses normative Moment in die Literatur ein: Wo Rousseau das philosophische Ideal einer marktfernen économie domestique im Rahmen eines Romans fiktional entfaltet, werden die noch bei Brunner wirksamen imaginären Anteile des 
"Haus«-Konzepts greifbar. Hier liegen die Aufgaben einer Literatur- und Kulturgeschichte des Oikonomischen. Die Ergebnisse der neueren Sozialgeschichte sind für eine Untersuchung des Imaginären insofern relevant, als sie mit Abstand zur Geschichte des zumeist normativ gebrauchten Begriffs die Realität häuslichen Lebens in den Blick rücken, einschließlich jener "hausgemachten « Traumata wie Sklavenarbeit und spätere Formen der Ausbeutung, die im Rahmen der Oikonomik nicht zur Sprache kommen.

Ohne es also auf die literarische Beglaubigung historischer Befunde abzusehen, ist vielmehr nach bestimmten literarischen Konstellationen und Imaginationsräumen zu fragen, in denen »das Haus nach seinem Ende « wiederkehrt. Drei unterschiedliche Bedeutungen des Worts können die Aspekte dieser Untersuchung verdeutlichen: So kann das »Haus " nämlich als Herrschaftseinheit betrachtet werden, als architektonische Einheit und schließlich als Korporation. Zunächst ist festzustellen, dass die oikonomische Herrschaftslehre im 18. Jahrhundert zunehmend in Konflikt geriet mit dem naturrechtlichen Gleichheitsideal. Das betraf nicht nur das Verhältnis zwischen dem Hausherren und den Bedienten. Dieser Konflikt zeichnete sich auch im Verhältnis der Geschlechter ab. Die Romanliteratur reagiert darauf mit fiktionalen Entwürfen einer neuen häuslichen Regimentalität. Im späteren 19. Jahrhundert ist zu beobachten, dass diese Frage am globalisierten Horizont völkerkundlichen Wissens in einer Gegenüberstellung von patriarchalischen und matriarchalischen Gesellschaften und mit Blick auf das »Männerhaus « verhandelt wird.

Hinsichtlich des Gebäudes ist bemerkenswert, dass der Volkskundler Riehl nicht nur das traditionelle Bauernhaus schildert, sondern ausführlich auch jene modernen Wohnhäuser, denen er es in modernitätskritischer Absicht entgegensetzt. Die detaillierte Beschreibung ihrer Räume entwirft das Bild eines gefährlich instabilen Gefüges, das sich auch in der literarischen Imagination als erstaunlich frequent erweist. Oftmals taucht eben dort, wo eine alte Gesamthäuslichkeit wiederhergestellt werden soll, die Vorstellung eines Hauses auf, das zerfällt und zu keiner dauerhaften räumlichen Struktur mehr geordnet werden kann. Auch hier ist ein Transfer zwischen Kulturgeschichtsschreibung und Literatur zu vermuten, finden sich ähnlich angstbesetzte Szenarien doch auch bei Schriftstellern wie Adalbert Stifter. Wohlgemerkt bleibt es hier keineswegs bei einer bloß nostalgischen Diagnose. Die zerfallenden Häuser, fehlenden Fundamente und geschrumpften Zimmer, von denen immer wieder erzählt wird, entfalten eine diskursive Produktivität und werden implizit vielfach zur Antriebskraft literarischen oder wissenschaftlichen Schreibens erklärt, etwa wenn Riehl die Kulturgeschichte in der Suche nach den verlorenen "Schätzen des Hauses « begründen möchte.

Ein Ende des Hauses droht nach Riehl über den materiellen Niedergang traditioneller Raumstrukturen hinaus hinsichtlich seiner korporativen Verfassung. Deren Besonderheit besteht darin, in sich naturnotwendige und freiwillige 
Beziehungen zu vereinen: »Durch das Absterben des Hauses, als der halb naturnotwendigen, halb freiwilligen Genossenschaft, ist ein Mittelglied zwischen der Familie und der Gesellschaftsgruppe verloren gegangen ${ }^{6}{ }^{6}$ Im Kontext von Riehls Ausführungen ist es unmissverständlich, welche Beziehungen als »naturnotwendige « bzw. "freiwillige « zu bezeichnen sind: Während familiale und verwandtschaftliche Beziehungen als naturnotwendig angesehen werden, sind Arbeits- und Dienstverhältnisse ihrem Wesen nach freiwillig. Dieses Kriterium der Freiwilligkeit als gesellschaftsbildender Kraft jenseits von naturnotwendigen Bindungen wird auch dort eingesetzt, wo es gilt, ausgehend vom Haus die grundlegenden Formen sozialer Vergemeinschaftungen und kultureller Evolutionen zu rekonstruieren. Schurtz etwa beschreibt in seiner um 1900 breit rezipierten Studie Altersklassen und Männerbünde die Herausbildung des »Männerhauses « als grundlegend für gesellschaftliche Entwicklungen. Dieser ethnologische Befund wird schließlich von Max Weber übernommen. Er bildet in Webers Werk den Ausgangspunkt zur Entwicklung des Charisma-Konzeptes, das als Gegensatz zur Autorität des Hausvaters gedacht wird ${ }^{7}$, ohne deshalb die Vorstellung einer häuslich organisierten Herrschaft preiszugeben; diese wird vielmehr durch das "Männerhaus « auf die Wirksamkeit eines symbolischen Ortes verpflichtet. Allerdings wird bereits bei Riehl die Kehrseite solcher Anthropologisierungen kultureller Befunde sichtbar: Anhand des Kriteriums der »Hausfähigkeit « wird eine Abspaltung der (Lumpen-)Proletarier von (ehrlichen) Arbeitern vollzogen. All jenen, die nicht in der Lage sind, sich häuslicher Kulturtechniken - wie etwa dem Aufstellen von Familienchroniken - zu bedienen oder zumindest als Knecht oder Magd Teil des ganzen Hauses zu werden, steht es frei auszuwandern.

An eben dieser Unterscheidung von Naturnotwendigkeit und Freiwilligkeit sollte sich eine Reihe von kulturtheoretischen und literarischen Überlegungen zum Haus abarbeiten. In einigen Fällen erfuhr sie dabei eine neue Besetzung. So argumentiert auch der Ethnologe Claude Lévi-Strauss, die Besonderheit des Hauses bestehe darin, sich nach den Regeln der Abstammung und der ehelichen und schwiegerverwandtschaftlichen Allianz perpetuieren zu können. ${ }^{8}$ Die eheliche Allianz folgt einer bewussten, aus der Perspektive des Hauses "freiwilligen " Wahl und vermag dadurch Automatismen und "natürliche « Abfolgen in der Abstammung zu bekräftigen, zu korrigieren oder außer Kraft zu setzen. Eine solche dynastische Kombinatorik wiederum ist nicht allein in außereuropäi-

\footnotetext{
${ }^{6}$ Riehl (Anm. 1), 156.

${ }^{7}$ Zum Antagonismus von 'Haus` und 'Charisma bei Weber vgl. Hans Derks, „Über die Faszination des 'Ganzen Hauses`“, Geschichte und Gesellschaft 22 (1996), 221-242, hier: 231f.

${ }^{8}$ Vgl. Claude Lévi-Strauss, "Stillstand und Geschichte. Plädoyer für eine Ethnologie der Turbulenzen ", in: Ulrich Raulff (Hrsg.), Vom Umschreiben der Geschichte, Berlin 1986, 68-87.
} 
schen »Häusergesellschaften " und in der Geschichte mittelalterlicher dynastischer Häuser aufzusuchen, sondern auch in den Häuserromanen des 19. Jahrhunderts wie etwa Theodor Fontanes Vor dem Sturm. Somit könnte - eine vorläufige Vermutung - die strukturale Verwandtschaftsethnologie LéviStrauss'scher Prägung viel stärker an die ihr unmittelbar vorangehende wilhelminische und viktorianische Häuserliteratur anzubinden sein als es bislang in der Geschichte der Sozialanthropologie geschehen ist.

Nacim Ghanbari, Saskia Haag, Marcus Twellmann (Siegen, Konstanz) 